\title{
Relationship Between Fibroblast Growth Factor 19 and Diabetic Retinopathy in Patients with Type 2 Diabetes Mellitus
}

\author{
Jin Ook Chung' \\ Seon-Young Park ${ }^{2}$ \\ Dong Hyeok Cho' \\ Dong Jin Chung' \\ Min Young Chung' \\ 'Division of Endocrinology and Metabolism, \\ Department of Internal Medicine, Chonnam \\ National University Medical School, \\ Gwangju, 50I-757, Republic of Korea; \\ ${ }^{2}$ Division of Gastroenterology and \\ Hepatology, Department of Internal \\ Medicine, Chonnam National University \\ Medical School, Gwangju, 50 I-757, Republic \\ of Korea
}

Correspondence: Jin Ook Chung; Min Young Chung

Division of Endocrinology and Metabolism, Department of Internal Medicine, Chonnam National University Medical School, 8 Hak-Dong, Dong-Gu, Gwangju, 50I-757, Republic of Korea Tel +82-62-220-6296; +82-62-220-6297 Fax +82-62-225-8578

Email imagine-jjo@hanmail.net; jrjjo222@gmail.com

\begin{abstract}
Aim: This study aimed to evaluate whether fibroblast growth factor 19 (FGF19) is associated with the risk of diabetic retinopathy in patients with type 2 diabetes mellitus (T2DM). Methods: A total of 357 patients with T2DM were investigated in this cross-sectional study. Logistic regression analysis was performed to assess the association between FGF19 level and diabetic retinopathy.
\end{abstract}

Results: Serum FGF19 level was significantly lower in patients with diabetic retinopathy in those without diabetic retinopathy. The multivariable analysis revealed a significant association between serum FGF19 level and diabetic retinopathy (odds ratio for every 1 standard deviation increase in logarithmic value $=0.69,95 \%$ confidence interval $0.51-0.94, p=$ 0.019).

Conclusion: Serum FGF19 level was negatively associated with diabetic retinopathy in patients with T2DM.

Keywords: diabetic retinopathy, fibroblast growth factor 19, type 2 diabetes mellitus

\section{Introduction}

Diabetic retinopathy is a common vision-threatening disease involving the retina in patients with diabetes mellitus. ${ }^{1}$ Apart from its influence on visual impairment, a large body of evidence has demonstrated that diabetic retinopathy is linked to increased risks of systemic vascular events leading to heart failure, coronary artery disease, and stroke, as well as increased mortality in patients with type 2 diabetes mellitus (T2DM). ${ }^{2,3}$ Moreover, diabetic retinopathy may reduce the quality of life and cause the functional impairments in patients with T2DM. ${ }^{4}$ Although extensive evidence indicates that chronic hyperglycemia predominantly contributes to the pathogenesis of diabetic retinopathy, it is implausible that diabetic retinopathy arises from hyperglycemia alone, and it is believed that more than a single factor may be involved in its pathogenesis. ${ }^{1}$

Fibroblast growth factor 19 (FGF19) is a newly identified member of the FGF family that lacks in a classic heparin-binding domain. ${ }^{5}$ Due to this characteristic, FGF19 is secreted into the bloodstream and has hormone-like effects through the activation of FGF receptors with beta-klotho as a cofactor, unlike other classic FGFs that function in a paracrine manner via a direct interaction with heparin/heparin sulfate. $^{5,6}$ Previous studies have reported a negative association between FGF19 level and cardiovascular disease risk. ${ }^{7,8}$ In experimental studies, FGF19 increased 
the energy expenditure and prevented the development of diabetes in rodent models. ${ }^{9,10}$ Recent clinical studies have demonstrated an inverse association between FGF19 level and the risk of diabetes. ${ }^{11,12}$ In addition, FGF19 was reported to have protective effects against retinal degeneration in other experimental studies. ${ }^{13,14}$ However, it remains unclear whether FGF19 plays a role in the development of diabetic retinopathy. To our knowledge, the relationship between FGF19 level and diabetic retinopathy in patients with T2DM has not been reported to date.

The purpose of this study was to investigate the relationship between FGF19 level and diabetic retinopathy in patients with T2DM.

\section{Methods}

\section{Patients}

This cross-sectional study included a total of 357 randomly chosen patients with T2DM who visited the diabetes clinic of our hospital between June 2017 and January 2019. The diagnosis of T2DM was made according to "Report of the Expert Committee on the Diagnosis and Classification of Diabetes Mellitus". 15 Patients with chronic liver diseases, biliary system disorders, thyroid dysfunction, infection or inflammatory disorders, use of glucocorticoids or bile acid sequestrants, advanced renal dysfunction (estimated glomerular filtration rate [eGFR] $<60 \mathrm{~mL} / \mathrm{min} / 1.73 \mathrm{~m}^{2}$ ), stroke, coronary artery disease, heart failure, or malignant disease were excluded. Clinical data on the duration of diabetes, medical history, and other health conditions were collected through standardized forms. Hypertension was defined as a blood pressure of $\geq 140 / 90 \mathrm{mmHg}$ or the use of antihypertensive drugs. Hyperlipidemia was defined as a total cholesterol level of $\geq 6.5 \mathrm{mmol} / \mathrm{L}$ and/or a triglyceride level of $\geq 2.3 \mathrm{mmol} / \mathrm{L}$, or the use of lipid-lowering agents. This study was approved by an ethics committee of Chonnam National University Hospital (No. CNUH2016-170). All participants provided written informed consent. The study was performed according to the Declaration of Helsinki guidelines.

\section{Measurements}

After an overnight fast, venous blood samples were collected from the patients. A lipid profile test, including triglycerides, high-density lipoprotein cholesterol, total cholesterol, and low-density lipoprotein cholesterol, was performed using an AU5400 device (Olympus,
Tokyo, Japan). We measured the fibrinogen level using a coagulation analyzer (Sysmex, IL, USA). The measurement of the glycated hemoglobin $\left(\mathrm{HbA}_{1 \mathrm{c}}\right)$ level was performed using an ion-exchange liquid chromatography system (Tosoh, Tokyo, Japan). We measured the serum FGF19 level by a sandwich enzyme-linked immunosorbent assay (ELISA) (FGF19 Quantikine ${ }^{\circledR}$ ELISA kit, R\&D Systems, Minneapolis, MN, USA), according to the instructions of the manufacturer. The inter-assay and intra-assay coefficients of variation were $6.5 \%$ and $5.4 \%$, respectively. We assessed eGFR using the Chronic Kidney Disease Epidemiology Collaboration equation. ${ }^{16}$ We estimated urinary albumin excretion based on the urinary albumin-to-creatinine ratio (UACR) in random urine samples. Fundus examination with pupil dilation was performed by an ophthalmologist to screen for diabetic retinopathy. The patients were classified into the no diabetic retinopathy, nonproliferative diabetic retinopathy (NPDR), and proliferative diabetic retinopathy (PDR) groups. NPDR or PDR was defined as diabetic retinopathy in this study. The internal validity of the grading process of retinal photographs was assessed in 64 randomly chosen cases by reclassifying them in a masked manner. The intrarater repeatability kappa value was 0.93 , implying good reliability.

\section{Statistical Analyses}

The sample size was determined to detect a medium effect size (d) of 0.5 with $\alpha$ of 0.05 and a power of $90 \%$. Using $\mathrm{G}^{*}$ Power 3.1.9.2, ${ }^{17}$ the sample size was computed for a 1:3 ratio of retinopathy/no retinopathy using a twotailed test. The estimated total sample size was 228 , and a minimum of 57 patients with diabetic retinopathy would detect a difference in FGF19 level.

The results are expressed as mean \pm standard deviation (SD) or median (interquartile range), unless otherwise indicated. The chi-square test was used to evaluate differences in categorical variables. The Mann-Whitney $U$-test or Student's $t$-test was used for comparisons between two groups, as appropriate. The patients were categorized based on serum FGF19 tertiles $(<101.3$, 101.3-178.3 and $\geq 178.3 \mathrm{pg} / \mathrm{mL}$ ). The analysis of variance (ANOVA) test or Kruskal-Wallis test was used to evaluate differences across the FGF19 tertiles for continuous variables.

To examine the association between FGF19 level and retinopathy, we performed multivariable analyses using logistic regression models with identified factors and formerly known risk factors. Data with skewed 
distributions were logarithmically transformed before the analysis. On account of the skewed distribution of FGF19 levels, the odds ratio (OR) was calculated for every 1 SD increase in the logarithmic value of FGF19 level. Data analyses were performed using SPSS 20.0 (SPSS, Chicago, IL, USA). For statistical tests, an $\alpha$ level of 0.05 was used.

\section{Results}

The characteristics of patients with T2DM are presented in Table 1. Patients with diabetic retinopathy had a longer duration of diabetes, higher $\mathrm{HbA}_{1 \mathrm{c}}$ level, higher UACR, higher fibrinogen level, and higher prevalence of insulin use than those without diabetic retinopathy. Serum FGF19 level was significantly lower in patients with diabetic retinopathy in those without diabetic retinopathy (104.7 [71.0-170.1] pg/ $\mathrm{mL}$ vs 131.1 [92.6-200.1], respectively; $p=0.010$ ) (Table 1). Serum FGF19 level was comparable between different stages of diabetic retinopathy $(98.6[69.4-155.0] \mathrm{pg} / \mathrm{mL}$ for NPDR [ $\mathrm{n}=53]$ vs 116.6 [77.6-196.2] $\mathrm{pg} / \mathrm{mL}$ for PDR groups $[\mathrm{n}=24]$, respectively; $p=0.262$ ).
The patients were classified into three groups according to serum FGF19 level. Table 2 shows the characteristics of the patients according to the FGF19 tertile. Body mass index decreased and LDL-C increased across FGF19 tertiles. The prevalence of diabetic retinopathy decreased with increasing tertiles of FGF19 level.

We evaluated the association between serum FGF19 level and diabetic retinopathy in patients with T2DM using logistic regression models (Table 3). The significant association between serum FGF19 level and diabetic retinopathy persisted (OR per 1 SD increase in logarithmic value $=0.69,95 \%$ CI $0.51-0.94, p=0.019)$ after adjusting for age, sex, body mass index, hypertension, hyperlipidemia, fibrinogen, $\mathrm{HbA}_{1 \mathrm{c}}$, diabetes duration, eGFR, UACR, and use of insulin and OHAs (model 2). Alternatively, when UACR treated as categorical variable (UACR $\geq 30 \mathrm{mg} / \mathrm{g}$ vs UACR $<30 \mathrm{mg} / \mathrm{g}$ ) was included as an independent variable in the model (model 2) instead of UACR treated as a continuous variable, serum FGG19 level was still associated with diabetic retinopathy (OR per $1 \mathrm{SD}$ increase in logarithmic value $=0.70,95 \%$ CI $0.52-0.95, p=0.020$ ).

Table I Characteristics of Patients with T2DM

\begin{tabular}{|c|c|c|c|}
\hline & Diabetic Retinopathy (-) & Diabetic Retinopathy (+) & p-value \\
\hline $\mathrm{n}$ & 280 & 77 & \\
\hline Age (years) & $56.8 \pm 11.3$ & $57.4 \pm 11.2$ & 0.690 \\
\hline Men (\%) & $142(50.7)$ & $4 \mid(53.2)$ & 0.694 \\
\hline Hypertension, n (\%) & $159(56.8)$ & $51(66.2)$ & 0.136 \\
\hline Hyperlipidemia, n (\%) & $167(59.6)$ & $44(57.1)$ & 0.693 \\
\hline Body mass index $\left(\mathrm{kg} / \mathrm{m}^{2}\right)$ & $26.2 \pm 4.2$ & $25.1 \pm 4.3$ & 0.040 \\
\hline Duration of diabetes (years) & $2.0(0.2-8.8)$ & II $.0(4.5-20.0)$ & $<0.001$ \\
\hline Systolic BP (mmHg) & $133.6 \pm 17.9$ & $134.6 \pm 15.8$ & 0.640 \\
\hline Diastolic BP (mmHg) & $78.3 \pm 12.1$ & $78.6 \pm 11.2$ & 0.847 \\
\hline $\mathrm{HbA}_{\mathrm{Ic}}(\%)$ & $7.5 \pm 1.7$ & $8.3 \pm 1.7$ & $<0.001$ \\
\hline $\mathrm{HbA}_{\mathrm{Ic}}(\mathrm{mmol} / \mathrm{mol})$ & $58 \pm 19$ & $67 \pm 18$ & $<0.001$ \\
\hline Triglycerides (mmol/L) & $1.3(1.0-2.0)$ & $1.4(1.0-2.0)$ & 0.976 \\
\hline LDL-C (mmol/L) & $2.6 \pm 0.8$ & $2.5 \pm 0.8$ & 0.113 \\
\hline HDL-C (mmol/L) & $1.3 \pm 0.3$ & $1.2 \pm 0.3$ & 0.434 \\
\hline Total cholesterol $(\mathrm{mmol} / \mathrm{L})$ & $4.4 \pm 1.1$ & $4.2 \pm 1.1$ & 0.158 \\
\hline UACR (mg/g Cr) & II.I (6.8-27.0) & $21.3(10.4-65.8)$ & $<0.001$ \\
\hline UACR $\geq 30 \mathrm{mg} / \mathrm{g} \mathrm{Cr}, \mathrm{n}$ (\%) & $64(22.9)$ & $31(40.3)$ & 0.002 \\
\hline eGFR $\left(\mathrm{mL} / \mathrm{min} / 1.73 \mathrm{~m}^{2}\right)$ & $98.1 \pm 14.1$ & $94.9 \pm 16.4$ & 0.090 \\
\hline Fibrinogen $(\mu \mathrm{mol} / \mathrm{L})$ & $8.6(7.6-10.0)$ & $9.2(8.3-10.5)$ & 0.016 \\
\hline FGFI9 (pg/mL) & |3I.I (92.6-200.I) & 104.7 (7I.0-I70.1) & 0.010 \\
\hline Use of OHAs, n (\%) & $166(59.3)$ & $43(55.8)$ & 0.587 \\
\hline Use of insulin, $n(\%)$ & $28(10.0)$ & $22(28.6)$ & $<0.001$ \\
\hline
\end{tabular}

Notes: Values are expressed as mean \pm standard deviation or median (interquartile range). Statistical analysis was performed using the Mann-Whitney U-test, Student's $t$-test, or the chi-square test.

Abbreviations: T2DM, type 2 diabetes mellitus; BP, blood pressure; HbA $1 \mathrm{c}$, glycated hemoglobin; LDL-C, low-density lipoprotein cholesterol; HDL-C, high-density lipoprotein cholesterol; UACR, urinary albumin-to-creatinine ratio; eGFR, estimated glomerular filtration rate; FGFI9, fibroblast growth factor 19; OHAs, oral hypoglycemic agents. 
Table 2 Characteristics of Patients with T2DM According to Serum FGFI9 Tertiles

\begin{tabular}{|c|c|c|c|c|}
\hline Serum FGFI 9 Level (pg/mL) & Tertile I (<98.5) & Tertile 2 (98.5-167.4) & Tertile $3(\geq 167.4)$ & $p$-value \\
\hline $\mathrm{n}$ & 119 & 119 & 119 & \\
\hline Age (years) & $55.3 \pm 11.6$ & $57.4 \pm 10.2$ & $58.1 \pm 11.8$ & 0.140 \\
\hline Men (\%) & $62(52.1)$ & $6 I(5 I .3)$ & $60(50.4)$ & 0.967 \\
\hline Hypertension, n (\%) & $70(58.8)$ & $66(55.5)$ & $74(62.2)$ & 0.574 \\
\hline Hyperlipidemia, n (\%) & $72(60.5)$ & $68(57.1)$ & 7I (59.7) & 0.860 \\
\hline Body mass index $\left(\mathrm{kg} / \mathrm{m}^{2}\right)$ & $27.0 \pm 4.4$ & $25.6 \pm 4.3$ & $25.4 \pm 3.9$ & 0.006 \\
\hline Duration of diabetes (years) & $4.0(0.4-11.0)$ & $4.0(0.2-10.0)$ & $3.0(0.2-10.0)$ & 0.831 \\
\hline Systolic BP (mmHg) & $132.8 \pm 14.7$ & $133.3 \pm 16.5$ & $135.3 \pm 20.6$ & 0.490 \\
\hline Diastolic BP $(\mathrm{mmHg})$ & $78.2 \pm 11.1$ & $77.9 \pm 11.0$ & $79.0 \pm 13.5$ & 0.788 \\
\hline $\mathrm{HbA}_{\mathrm{Ic}}(\%)$ & $7.7 \pm 1.6$ & $7.9 \pm 2.0$ & $7.4 \pm 1.6$ & 0.056 \\
\hline $\mathrm{HbA}_{\mathrm{Ic}}(\mathrm{mmol} / \mathrm{mol})$ & $60 \pm 17$ & $63 \pm 22$ & $57 \pm 17$ & 0.056 \\
\hline Triglycerides (mmol/L) & $1.4(1.0-2.0)$ & $1.3(1.0-2.0)$ & $1.3(1.0-2.0)$ & 0.822 \\
\hline LDL-C (mmol/L) & $2.5 \pm 0.7$ & $2.6 \pm 0.8$ & $2.7 \pm 0.9$ & 0.036 \\
\hline HDL-C (mmol/L) & $1.3 \pm 0.4$ & $1.3 \pm 0.3$ & $1.3 \pm 0.3$ & 0.712 \\
\hline Total cholesterol $(\mathrm{mmol} / \mathrm{L})$ & $4.2 \pm 1.0$ & $4.4 \pm 1.1$ & $4.5 \pm 1.2$ & 0.055 \\
\hline UACR (mg/g Cr) & $11.9(7.0-29.8)$ & $\mid 2.6(7.2-3 \mid .2)$ & I3.7 (7.3-43.5) & 0.807 \\
\hline $\mathrm{UACR} \geq 30 \mathrm{mg} / \mathrm{g} \mathrm{Cr}, \mathrm{n}(\%)$ & $29(24.4)$ & $31(26.1)$ & $35(29.4)$ & 0.669 \\
\hline eGFR $\left(\mathrm{mL} / \mathrm{min} / 1.73 \mathrm{~m}^{2}\right)$ & $98.2 \pm 15.7$ & $97.8 \pm 13.2$ & $96.1 \pm 15.0$ & 0.490 \\
\hline Fibrinogen $(\mu \mathrm{mol} / \mathrm{L})$ & $8.8(7.7-10.2)$ & $8.6(7.7-9.9)$ & $9.1(7.8-10.4)$ & 0.255 \\
\hline FGFI9 (pg/mL) & $74.1(59.6-90.1)$ & $122.0(|||.8-| 43.5)$ & $245.1(197.6-318.5)$ & $<0.001$ \\
\hline Diabetic retinopathy, n (\%) & $35(29.4)$ & $23(19.3)$ & $19(16.0)$ & 0.032 \\
\hline Use of OHAs, n (\%) & $77(64.7)$ & $60(50.4)$ & $72(60.5)$ & 0.071 \\
\hline Use of insulin, n (\%) & $20(16.8)$ & $17(14.3)$ & $13(10.9)$ & 0.423 \\
\hline
\end{tabular}

Notes: Values are expressed as mean \pm standard deviation or median (interquartile range). Statistical analysis was performed using the analysis of variance test, KruskalWallis test, or the chi-square test.

Abbreviations: T2DM, type 2 diabetes mellitus; BP, blood pressure; $\mathrm{HbA}_{\mathrm{Ic}}$, glycated hemoglobin; LDL-C, low-density lipoprotein cholesterol; HDL-C, high-density lipoprotein cholesterol; UACR, urinary albumin-to-creatinine ratio; eGFR, estimated glomerular filtration rate; FGFI9, fibroblast growth factor 19; OHAs, oral hypoglycemic agents.

\section{Discussion}

We investigated the association between serum FGF19 level and diabetic retinopathy in patients with T2DM in this study. After adjustment for conventional risk factors including $\mathrm{HbA}_{1 \mathrm{c}}$, duration of diabetes, and hypertension, serum FGF19 level was significantly and negatively associated with diabetic retinopathy, although it was not correlated with the severity of diabetic retinopathy.

Table 3 Logistic Regression Model of the Association Between FGFI9 Level and Diabetic Retinopathy in Patients with T2DM

\begin{tabular}{|l|c|c|c|}
\hline \multirow{2}{*}{\begin{tabular}{l} 
WGF $^{+}{ }^{\dagger}$ (pgl \\
\cline { 2 - 4 }
\end{tabular}} & $\begin{array}{c}\text { Odd } \\
\text { Ratio }\end{array}$ & $\begin{array}{c}\text { 95\% Confidence } \\
\text { Interval }\end{array}$ & P-value \\
\hline Unadjusted & 0.75 & $0.58-0.97$ & 0.030 \\
Model I & 0.70 & $0.54-0.92$ & 0.010 \\
Model 2 & 0.69 & $0.51-0.94$ & 0.019 \\
\hline
\end{tabular}

Notes: 'Values were logarithmically transformed before the analysis. Model I: adjusted for age, sex, and body mass index. Model 2: model I plus hypertension, fibrinogen ${ }^{\dagger}$, hyperlipidemia, glycated hemoglobin, diabetes duration ${ }^{\dagger}, \mathrm{eGFR}, \mathrm{UACR}^{\dagger}$, and use of insulin and oral hypoglycemic agents.

Abbreviations: FGFI9, fibroblast growth factor 19; T2DM, type 2 diabetes mellitus.
Recently, FGF19 has gained attention as a metabolic regulator. ${ }^{6}$ Although the production of FGF19 largely occurs in the epithelium of the small intestine, it is expressed in nearly all tissues. ${ }^{5}$ As FGF19 lacks a heparinbinding domain, it is considered to act through the circulation. $^{5}$ A growing body of evidence shows that FGF19 functions as an extracellular signaling molecule that plays a role in diverse biological functions, including bile acid metabolism and gallbladder filling. ${ }^{6}$ In addition, previous studies have reported a negative association between FGF19 level and cardiovascular disease risk. ${ }^{7,18,19}$ Hao et $\mathrm{al}^{8}$ observed decreased levels of serum FGF19 in patients with coronary heart disease and that serum FGF19 level was inversely associated with the severity of coronary lesions, suggesting its usefulness as a potential marker of cardiovascular diseases.

Recent studies indicated a strong link between FGF19 and the regulation of glucose metabolism. ${ }^{11}$ In animal models, transgenic overexpression of FGF19 or administration of exogenous FGF19 improved insulin sensitivity and glucose tolerance. ${ }^{9,10}$ Patients with gestational diabetes mellitus were 
reported to have lower serum FGF19 levels than healthy pregnant women. ${ }^{20}$ Reduced levels of serum FGF19 were also found in patients with T2DM compared with those without T2DM. ${ }^{11,12,19}$ Fang et al ${ }^{11}$ reported that a decreased serum FGF19 level was associated with worsening of the glucometabolic status, from normal glucose tolerance to T2DM. FGF19 is involved in the development of ocular tissue. ${ }^{14}$ Chromosome mapping has shown that FGF19 is located at chromosome 11q13.1, a region associated with osteoporosis-pseudoglioma syndrome characterized by skeletal and retinal defects. ${ }^{21}$ In addition, FGF19 has been reported to have beneficial effects against retinal degeneration in experimental studies. ${ }^{13}$ However, the associations between FGF19 and diabetic retinopathy remain unclear to date. In this study, we found that serum FGF19 level was significantly and inversely associated with diabetic retinopathy in patients with T2DM after adjusting for conventional risk factors for diabetic retinopathy. FGF19 has been reported to be associated with glycemic control and body mass index. ${ }^{7,11,18}$ Previous studies have also shown that FGF19 level is related to serum lipid profile, in line with the findings in the present study. ${ }^{22}$ Consequently, it might be assumed that these aspects partly explain the observed association between FGF19 level and diabetic retinopathy. ${ }^{1}$ The multivariable analysis, however, showed that the statistically significant relationship between FGF19 level and diabetic retinopathy persisted after adjustment for confounders, including body mass index, $\mathrm{HbA}_{1 \mathrm{c}}$, duration of diabetes, and hyperlipidemia. Accordingly, the results indicate that these factors did not significantly exert an influence on the association between FGF19 and diabetic retinopathy.

In addition, we note that the association between albuminuria and diabetic retinopathy has been reported in several studies of patients with T2DM. ${ }^{1,23}$ In accordance with the previous findings, ${ }^{1,23}$ our study showed that patients with diabetic retinopathy had higher UACR than those without diabetic retinopathy. Therefore, albuminuria might affect the findings observed in the present study. However, our study showed that the statistically significant relationship between FGF19 level and diabetic retinopathy was persistent after adjustment for UACR treated as continuous or categorical variable in the multivariable analysis. This suggests that the association between FGF19 and diabetic retinopathy is independent of albuminuria status. However, further studies are needed in this regard.

Even though the mechanisms underlying the association between FGF19 level and diabetic retinopathy remain unclear, there is a plausible explanation for the link between these two factors. FGF19 is related to oxidative stress, inflammation, and immune response, ${ }^{24-27}$ which are also cardinal contributors to the pathogenesis of diabetic retinopathy. ${ }^{1}$ Thus, these common mechanisms may explain the close relationship between FGF19 level and diabetic retinopathy. Further studies are needed to investigate the underlying mechanisms.

In the present study, patients without diabetic retinopathy had increased level of serum FGF19 compared with those with diabetic retinopathy. FGF19 level was comparable between NPDR and PDR subgroups. Thus, we speculate that FGF19 might play a pathophysiological role in early stages of diabetic retinopathy in patients with T2DM; however, this hypothesis is necessary to be verified by large-scale longitudinal studies.

Fibrinogen, a glycoprotein generated in the liver, is a crucial component in the coagulation cascade. ${ }^{28}$ In addition, a large body of evidence has demonstrated that fibrinogen is involved in inflammation. ${ }^{28}$ Fibrinogen is considered to regulate the inflammatory processes by binding to its receptor on immune cells. ${ }^{28}$ Increased fibrinogen levels indicate a pro-inflammatory status. ${ }^{28}$ Accumulating evidence has shown that inflammation is involved in the pathogenesis of diabetic retinopathy. ${ }^{29}$ In the current study, multivariable analysis showed that the relationship between FGF19 and diabetic retinopathy remained significant after adjusting for covariates including fibrinogen level. Consequently, these findings imply that mechanisms independent of fibrinogen level may be involved. ${ }^{30}$ However, further studies are needed because, in addition to inflammatory stimulants, other factors may have an impact on fibrinogen level.

This study has some limitations. Because of the crosssectional design, causal relationships between FGF19 level and diabetic retinopathy could not be inferred. In the present study, NPDR might be in remission in some patients when retinal examinations were performed. Thus, these patients might be classified as no diabetic retinopathy group in this study. If these patients were to be defined as NPDR group, diabetic retinopathy might be underestimated in such a case. However, because this would bias towards null, we believe that our results would not be significantly influenced. Although patients with coronary artery disease were excluded in the present study, patients with subclinical coronary artery disease might be included because coronary artery disease was diagnosed based on medical history and electrocardiogram. In addition, patients without advanced renal 
dysfunction were included in our study, and further studies are necessary to investigate whether our results might apply to T2DM patients with advanced renal dysfunction. Another limitation is that the sample size in this study is relatively small. However, despite the limitations above, we believe that the present study provides important information regarding relationships between FGF19 and diabetic retinopathy. Further large longitudinal studies are required to better assess the associations between FGF19 and diabetic retinopathy in patients with T2DM.

\section{Conclusion}

In conclusion, we found an inverse association between serum FGF19 level and diabetic retinopathy, although serum FGF19 level was not correlated with the severity of diabetic retinopathy. Further investigations are required to ascertain the mechanisms governing the relationship between FGF19 level and diabetic retinopathy.

\section{Acknowledgments}

This study was supported by Chonnam National University Hospital Biomedical Research Institute (BCRI20014).

\section{Disclosure}

The authors report no conflicts of interest in this work.

\section{References}

1. Cheung N, Mitchell P, Wong TY. Diabetic retinopathy. Lancet. 2010;376(9735):124-136. doi:10.1016/S0140-6736(09)62124-3

2. Cheung N, Wong TY. Diabetic retinopathy and systemic vascular complications. Prog Retin Eye Res. 2008;27(2):161-176. doi:10.1016/j.preteyeres.2007.12.001

3. Drinkwater JJ, Davis TME, Hellbusch V, Turner AW, Bruce DG, Davis WA. Retinopathy predicts stroke but not myocardial infarction in type 2 diabetes: the Fremantle diabetes study phase II. Cardiovasc Diabetol. 2020;19(1):43. doi:10.1186/s12933-020-01018-3

4. Brown MM, Brown GC, Sharma S, Shah G. Utility values and diabetic retinopathy. Am J Ophthalmol. 1999;128(3):324-330. doi:10.1016/ S0002-9394(99)00146-4

5. Itoh N, Ornitz DM. Fibroblast growth factors: from molecular evolution to roles in development, metabolism and disease. $J$ Biochem. 2011;149(2):121-130. doi:10.1093/jb/mvq121

6. Jahn D, Rau M, Hermanns HM, Geier A. Mechanisms of enterohepatic fibroblast growth factor 15/19 signaling in health and disease. Cytokine Growth Factor Rev. 2015;26(6):625-635. doi:10.1016/j.cytogfr.20 15.07.016

7. Stejskal D, Karpisek M, Hanulova Z, Stejskal P. Fibroblast growth factor-19: development, analytical characterization and clinical evaluation of a new ELISA test. Scand J Clin Lab Invest. 2008;68 (6):501-507. doi:10.1080/00365510701854967

8. Hao Y, Zhou J, Zhou M, et al. Serum levels of fibroblast growth factor 19 are inversely associated with coronary artery disease in Chinese individuals. PLoS One. 2013;8(8):e72345. doi:10.1371/journal. pone. 0072345
9. Tomlinson E, Fu L, John L, et al. Transgenic mice expressing human fibroblast growth factor-19 display increased metabolic rate and decreased adiposity. Endocrinology. 2002;143(5):1741-1747. doi:10.1210/endo.143.5.8850

10. Fu L, John LM, Adams SH, et al. Fibroblast growth factor 19 increases metabolic rate and reverses dietary and leptin-deficient diabetes. Endocrinology. 2004;145(6):2594-2603. doi:10.1210/ en.2003-1671

11. Fang Q, Li H, Song Q, et al. Serum fibroblast growth factor 19 levels are decreased in Chinese subjects with impaired fasting glucose and inversely associated with fasting plasma glucose levels. Diabetes Care. 2013;36(9):2810-2814. doi:10.2337/dc12-1766

12. Gerhard GS, Styer AM, Wood GC, et al. A role for fibroblast growth factor 19 and bile acids in diabetes remission after Roux-en-Y gastric bypass. Diabetes Care. 2013;36(7):1859-1864. doi:10.2337/dc12-2255

13. Siffroi-Fernandez S, Felder-Schmittbuhl MP, Khanna H, Swaroop A, Hicks D. FGF19 exhibits neuroprotective effects on adult mammalian photoreceptors in vitro. Invest Ophthalmol Vis Sci. 2008;49 (4):1696-1704. doi:10.1167/iovs.07-1272

14. Somm E, Jornayvaz FR. Fibroblast growth factor 15/19: from basic functions to therapeutic perspectives. Endocr Rev. 2018;39 (6):960-989. doi:10.1210/er.2018-00134

15. Expert Committee on the Diagnosis and Classification of Diabetes Mellitus. Report of the expert committee on the diagnosis and classification of diabetes mellitus. Diabetes Care. 2003;26(Suppl 1):S5-20. doi:10.2337/diacare.26.11.3160

16. Levey AS, Stevens LA, Schmid $\mathrm{CH}$, et al. A new equation to estimate glomerular filtration rate. Ann Intern Med. 2009;150 (9):604-612. doi:10.7326/0003-4819-150-9-200905050-00006

17. Faul F, Erdfelder E, Lang AG, Buchner A. G*Power 3: a flexible statistical power analysis program for the social, behavioral, and biomedical sciences. Behav Res Methods. 2007;39(2):175-191. doi:10.3758/BF03193146

18. Barutcuoglu B, Basol G, Cakir Y, et al. Fibroblast growth factor-19 levels in type 2 diabetic patients with metabolic syndrome. Ann Clin Lab Sci. 2011;41(4):390-396.

19. Liu WS, Tang MJ, Xu TL, et al. Association of serum fibroblast growth factor 19 levels with arteriosclerosis parameters assessed by arterial stiffness and atherogenic index of plasma in patients with type 2 diabetes. Diabetol Metab Syndr. 2020;12:44. doi:10.1186/ s13098-020-00552-0

20. Wang D, Zhu W, Li J, An C, Wang Z. Serum concentrations of fibroblast growth factors 19 and 21 in women with gestational diabetes mellitus: association with insulin resistance, adiponectin, and polycystic ovary syndrome history. PLoS One. 2013;8(11):e81190. doi:10.1371/journal.pone. 0081190

21. Xie MH, Holcomb I, Deuel B, et al. FGF-19, a novel fibroblast growth factor with unique specificity for FGFR4. Cytokine. 1999;11 (10):729-735. doi:10.1006/cyto.1999.0485

22. Dolegowska K, Marchelek-Mysliwiec M, Nowosiad-Magda M, Slawinski M, Dolegowska B. FGF19 subfamily members: FGF19 and FGF21. J Physiol Biochem. 2019;75(2):229-240. doi:10.1007/ s13105-019-00675-7

23. Rani PK, Raman R, Gupta A, Pal SS, Kulothungan V, Sharma T. Albuminuria and diabetic retinopathy in type 2 diabetes mellitus Sankara Nethralaya diabetic retinopathy epidemiology and molecular genetic study (SN-DREAMS, report 12). Diabetol Metab Syndr. 2011;3(1):9. doi:10.1186/1758-5996-3-9

24. Guo A, Li K, Xiao Q. Fibroblast growth factor 19 alleviates palmitic acid-induced mitochondrial dysfunction and oxidative stress via the AMPK/PGC-1alpha pathway in skeletal muscle. Biochem Biophys Res Commun. 2020;526(4):1069-1076. doi:10.1016/j.bbrc.2020.04.002

25. Fang Y, Zhao Y, He S, et al. Overexpression of FGF19 alleviates hypoxia/reoxygenation-induced injury of cardiomyocytes by regulating GSK-3beta/Nrf2/ARE signaling. Biochem Biophys Res Commun. 2018;503(4):2355-2362. doi:10.1016/j.bbrc.2018.06.161 
26. You M, Zhou Z, Daniels M, Jogasuria A. Endocrine adiponectin-FGF15/19 axis in ethanol-induced inflammation and alcoholic liver injury. Gene Expr. 2018;18(2):103-113. doi:10.3727/ 105221617X15093738210295

27. Gadaleta RM, Garcia-Irigoyen O, Cariello M, et al. Fibroblast growth factor 19 modulates intestinal microbiota and inflammation in presence of Farnesoid X receptor. EBioMedicine. 2020;54:102719. doi:10.1016/j.ebiom.2020.102719
28. Davalos D, Akassoglou K. Fibrinogen as a key regulator of inflammation in disease. Semin Immunopathol. 2012;34(1):43-62. doi:10.1007/s00281-011-0290-8

29. Tang J, Kern TS. Inflammation in diabetic retinopathy. Prog Retin Eye Res. 2011;30(5):343-358. doi:10.1016/j.preteyeres.2011.05.002

30. Greco M, Chiefari E, Accattato F, et al. MicroRNA-1281 as a novel circulating biomarker in patients with diabetic retinopathy. Front Endocrinol (Lausanne). 2020;11:528. doi:10.3389/fendo.2020.00528

\section{Publish your work in this journal}

Diabetes, Metabolic Syndrome and Obesity: Targets and Therapy is an international, peer-reviewed open-access journal committed to the rapid publication of the latest laboratory and clinical findings in the fields of diabetes, metabolic syndrome and obesity research. Original research, review, case reports, hypothesis formation, expert opinion and commentaries are all considered for publication. The manuscript management system is completely online and includes a very quick and fair peer-review system, which is all easy to use. Visit http://www.dovepress.com/testimonials.php to read real quotes from published authors. 